\title{
Regulation of angiopoietin-like protein 8 expression under different nutritional and metabolic status
}

\author{
Chang Guo ${ }^{1)}$, Zhicong Zhao ${ }^{1)}$, Xia Deng ${ }^{1)}$, Zian $\mathrm{Chen}^{2)}$, Zhigang $\mathrm{Tu}^{2)}$ and Guoyue Yuan ${ }^{1)}$ \\ 1) Department of Endocrinology, Affiliated Hospital of Jiangsu University, Zhenjiang, Jiangsu 212001, China \\ 2) Institute of Life Sciences, Jiangsu University, Zhenjiang, Jiangsu 212013, China
}

\begin{abstract}
Type 2 diabetes mellitus (T2DM) is a chronic metabolic disease with increasing prevalence worldwide. Angiopoietin-like protein 8 (ANGPTL8), a member of the angiopoietin-like protein family, is involved in glucose metabolism, lipid metabolism, and energy homeostasis and believed to be associated with T2DM. Expression levels of ANGPTL8 are often significantly altered in metabolic diseases, such as non-alcoholic fatty liver disease (NAFLD) and diabetes mellitus. Studies have shown that ANGPTL8, together with other members of this protein family, such as angiopoietin-like protein 3 (ANGPTL3) and angiopoietin-like protein 4 (ANGPTL4), regulates the activity of lipoprotein lipase (LPL), thereby participating in the regulation of triglyceride related lipoproteins (TRLs). In addition, members of the angiopoietin-like protein family are varyingly expressed among different tissues and respond differently under diverse nutritional and metabolic status. These findings may provide new options for the diagnosis and treatment of diabetes, metabolic syndromes and other diseases. In this review, the interaction between ANGPTL8 and ANGPTL3 or ANGPTL4, and the differential expression of ANGPTL8 responding to different nutritional and metabolic status during the regulation of LPL activity were reviewed.
\end{abstract}

Key words: Angiopoietin-like protein 8 (ANGPTL8), Diabetes mellitus, Metabolic status, Nutritional status

\section{Introduction}

Triglycerides (TGs) are one of the most important substrates for energy supply. Compared to carbohydrates and proteins, TGs have lower metabolic cost $[1,2]$. Therefore, synthesis and storage of TGs are particularly important to maintain the body's energy balance. Lipoprotein lipase (LPL) is a key rate-limiting enzyme in catalyzing hydrolysis of circulating triglyceride in chylomicrons $(\mathrm{CM})$ and very-low-density lipoprotein (VLDL), the major forms of triglycerides in plasma [3, 4].

LPL hydrolyzes TG from chylomicron and VLDL into free fatty acids, which are absorbed by surrounding tissues such as fat, muscle, and heart [5]. To cope with the energy requirement of the body under different nutritional and metabolic status, TGs in the circulation are mainly processed by LPL and distributed in oxidized tissues (such as muscle) and white adipose tissue (WAT)

Submitted Jul. 18, 2019; Accepted Sep. 10, 2019 as EJ19-0263 Released online in J-STAGE as advance publication Oct. 19, 2019 Correspondence to: Guoyue Yuan, Department of Endocrinology, Affiliated Hospital of Jiangsu University, 438 Jiefang Road, Zhenjiang, Jiangsu 212001, China.

E-mail: yuanguoyue@ujs.edu.cn

Correspondence to: Zhigang Tu, Institute of Life Sciences, Jiangsu University, 301 Xuefu Road, Jingkou District, Zhenjiang, Jiangsu 212013, China.

E-mail: zhigangtu@ujs.edu.cn under capillary endothelial cells [6]. LPL has hydrolytic activity in the form of dimer, and the dissociation of LPL dimer is the key to its spontaneous inactivation [7]. In the fasted state, LPL activity in WAT is down-regulated, while its activity in heart and skeletal muscle is upregulated to meet the energy needs of the body. In the fed state, LPL activity in WAT is increased, thereby increasing the energy reserve of the body $[4,7]$. Therefore, LPL plays an important role in maintaining the energy balance of the body.

Angiopoietin-like proteins (ANGPTLs) are a family of secreted glycoproteins consisting of the N-terminal helix domain and the fibrinogen-like C-terminal domain. ANGPTLs often participate in angiogenesis, stem cell expansion, inflammation, tissue remodeling, and lipid metabolism. Genetic and functional studies have shown that ANGPTL3, ANGPTL4, and ANGPTL8 play important roles in the regulation of LPL activity [8-12].

ANGPTL8 (also called RIFL, lipasin, and betatrophin) consists of 198 amino acids and is about $22 \mathrm{kDa}$ in size. The human ANGPTL8 gene was designated as LOC55908, C19ORF80, or TD26, and located at chromosome 19p13.2. In mice, the gene is located at chromosome 9 and is called Gm6484 or EG624219 [13]. Studies have shown that human ANGPTL8 is dominantly expressed in liver, while mouse ANGPTL8 has highest expression levels in liver and white and brown adipose tissues. ANGPTL8 is considered to be an atypical mem- 
ber of the ANGPTL protein family. Different from other members of the family, ANGPTL8 has a characteristic $\mathrm{N}$-terminal domain which is required for inhibition of LPL activity but lacks the C-terminal domain [14-16]. Some studies show that ANGPTL3 and ANGPTL4 are the most similar family members of ANGPTL8 [17]. ANGPTL8 plays its physiological roles through PI3K, Akt, FOXO1 and other signaling pathways [13, 18-20]. Many studies have confirmed that ANGPTL8 is closely related to the metabolic status of the body, and is associated with glucose and lipid metabolism as well as the occurrence of insulin resistance [13, 19, 21, 22]. The transcription and translation of ANGPTL8 are regulated by many factors, such as glucose, insulin and GLP-1 [13, 18-20]. The expression levels of ANGPTL8 is also regulated by the nutritional status, and its expression is significantly increased in the refeeding state [23].

\section{ANGPTL8 in Regulation of Lipid Metabolism}

ANGPTL8 is regulated by the nutritional status of the body and has been studied primarily as a new factor involved in lipid metabolism. ANGPTL8 is mainly involved in the regulation of triglyceride levels by affecting LPL activity $[13,21,22]$.

\section{Evidence of ANGPTL8 regulating lipid metabolism}

Ren et al. discovered that during differentiation of mouse and human primary preadipocytes, the transcriptional levels of ANGPTL8 increased significantly with lipid droplet formation, along with changes of biomarkers of adipocyte differentiation, such as PPAR- $\gamma$, SCD1, FABP4, PNPLA2, etc [13]. Moreover, knocking down ANGPTL8 results in a decrease in lipid content in adipocytes [24]. TNF- $\alpha$ is a cytokine that inhibits adipogenesis and dedifferentiates adipocytes, and down-regulates the expression of biomarkers for adipose differentiation. Studies have shown that during the culture of precursor fat cells, the addition of TNF- $\alpha$ can significantly downregulate the expression of ANGPTL8 [25]. In addition, ANGPTL8 is also involved in lipolysis [26]. Some drugs that promote lipolysis, such as db-cAMP, forskolin, and isoproterenol, can significantly reduce the transcription levels of ANGPTL8. Loss of ANGPTL8 leads to a decrease in free fatty acids (FFAs). Consistently, the expression of genes involved in lipolysis (ANGPTL4 and leptin) and FA oxidation-related genes (Cpt1a, Cpt1b, and Pgc-1a) increases after knockdown of ANGPTL8 [13]. By using adenoviral vectors to overexpress ANGPTL8 or directly treating with recombinant ANGPTL8 protein, Zhang et al. found that ANGPTL8 can inhibit LPL activity and reduce triglyceride clear- ance in mice, thereby increasing serum triglyceride levels [21]. These results indicate that ANGPTL8 may play an important role in regulating functions of fat cells and lipid metabolism through affecting LPL activity.

\section{Interaction between ANGPTL3 and ANGPTL8}

ANGPTL3 is a hepatogenic secreted protein which also participates in lipid metabolism by regulating LPL activity. But the inhibitory effect of ANGPTL3 on LPL activity is not so strong that it can only play a role when its concentration is much larger than its physiological one [27]. Consistently, Chi et al. found that by injecting ANGPTL3 adenovirus, triglyceride levels in plasma in wild-type mice can be increased. The elevated levels of triglycerides are positively correlated with the amounts of adenovirus injected [28]. This indicates that ANGPTL3 indeed participates in the regulation of LPL activity in a dose-dependent manner.

Studies have shown that ANGPTL3 relies on ANGPTL8 to play a regulatory role in lipid metabolism. In the presence of ANGPTL8, the minimum amount of ANGPTL 3 was only 2 times of physiological concentration to significantly inhibit LPL activity [28]. On the contrary, injection of ANGPTL3 adenovirus into ANGPTL8 knockout mice did not significantly affect levels of triglyceride. This suggests that the mechanism by which ANGPTL3 regulates LPL activity may require the participation of ANGPTL8. ANGPTL8 can bind to the N-terminal domain of ANGPTL3 protein and facilitates cleavage of ANGPTL3 by exposing its cleavage site or recruiting relevant proteases. After the N-terminal domain is cleaved, the remaining part of ANGPTL3 is still bound to ANGPTL8 to form complexes which regulate LPL activity. In addition, Chi et al. also suggested that ANGPTL3 and ANGPTL8 can form complexes at different stoichiometric ratios, and the formation of such complexes occurs intracellularly, rather than outside of the cells after their secretion [28]. In fact, ANGPTL8 is rarely secreted extracellularly after intracellular synthesis. Although ANGPTL3 and ANGPTL8 do not have a fixed stoichiometric ratio when forming a complex, the ratio for a complex to exert the maximal LPL inhibition is approximately 1:1 [29].

Haller's group found that the C-terminus of ANGPTL8 protein has a short helix structure that covers its binding site, so ANGPTL8 alone cannot inhibit LPL activity (Fig. 1). When ANGPTL3 is present, this binding site will be opened and the inhibitory role of ANGPTL8 becomes effective. Therefore, in traditional opinion, ANGPTL3 plays a primary role in the ANGPTL3/ANGPTL8 complex [10]. However, an ANGPTL3/8 complex with a variant of ANGPTL3 that lacks LPL inhibitory activity still inhibited LPL activity. 
A

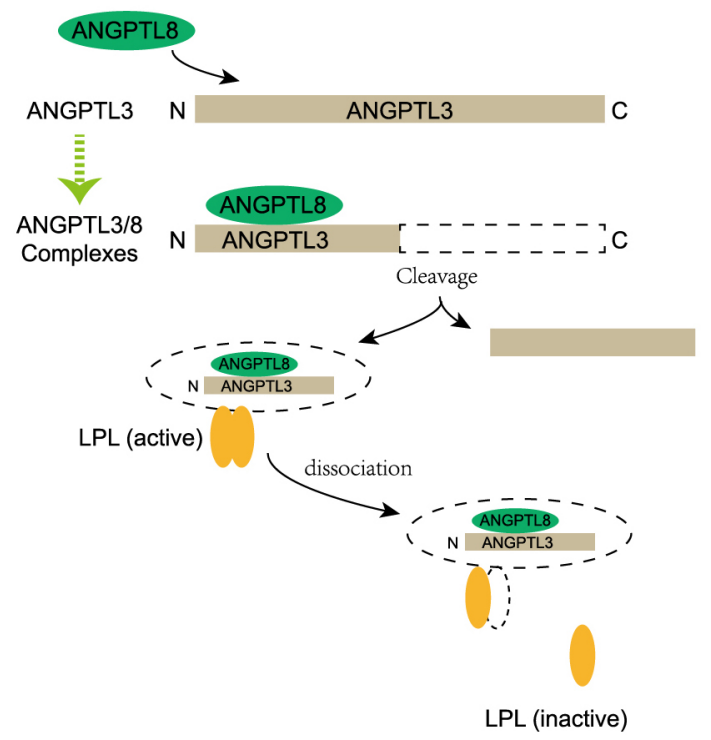

B

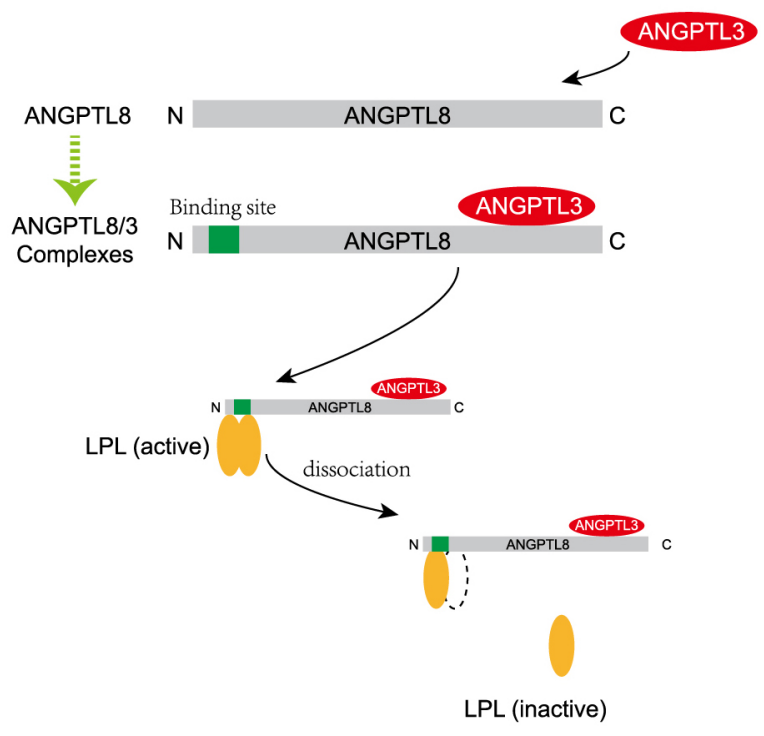

Fig. 1 Interaction between ANGPTL3 and ANGPTL8. A. The traditional view of the interaction between ANGPTL3 and ANGPTL8 that ANGPTL3 plays a primary role. ANGPTL8 binds to the N-terminal of ANGPTL3 which leads to the cleavage of ANGPTL3, and the remaining N-terminal of ANGPTL3 plays an active role. B. The novel view of the interaction between ANGPTL3 and ANGPTL8 that ANGPTL8 plays a primary role. ANGPTL3 binds to the C terminal of ANGPTL8, exposing the N-terminal site of ANGPTL8, which exerts the active role.

These results suggested that the activity of ANGPTL3 is not required for activity of the ANGPTL8/ANGPTL3 complex and the major inhibitory activity may be provided by ANGPTL8 [30]. In addition, a specific ANGPTL8 antibody that targets the C-terminus of ANGPTL8 without affecting the formation of the ANGPTL3/ANGPTL8 complex was used. This antibody can inactivate ANGPTL8, thereby reversing the inhibitory effects of the complex on lipoprotein lipase [28]. Therefore, they believe that ANGPTL8 may exert a major inhibitory effect on LPL activity in the ANGPTL3/ANGPTL8 complex.

It is well accepted that compared to their respective inhibitory effects of ANGPTL3 and ANGPTL8, the ANGPTL3/ANGPTL8 complex has greater inhibitory effects on LPL activity.

\section{Interaction between ANGPTL4 and ANGPTL8}

ANGPTL4 is a member of the ANGPTL protein family with approximately $50 \mathrm{kD}$ in size. In mice, ANGPTL4 is mainly expressed in white and brown adipose tissues [31, 32]. Studies have shown that the Nterminal helix domain of ANGPTL4 can bind to LPL and change it from active dimers to form inactive monomers [33]. In addition, ANGPTL4 can also reduce the secretion of LPL in adipocytes [34-36].

Previous studies have suggested that ANGPTL4 exert its function in a homogeneous dimer linked by disulfide bonds [37]. Recent studies by Mysling's group have

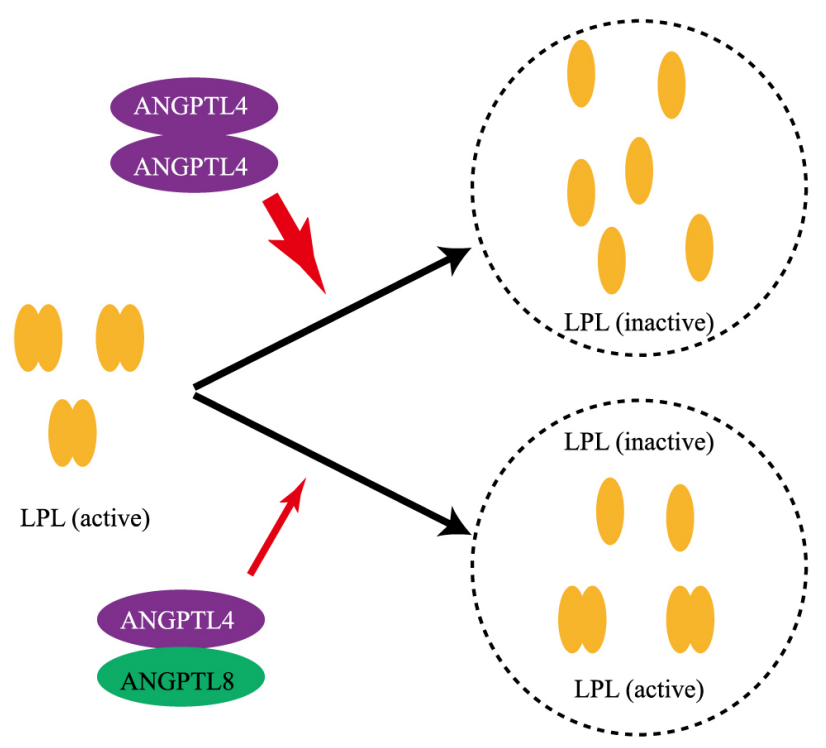

Fig. 2 ANGPTL8 and ANGPTL4 form a complex that inhibits LPL dissociation.

shown that, similar to ANGPTL3, ANGPTL4 can also form a complex with ANGPTL8 (Fig. 2). However, the activity of ANGPTL4/8 complex to inhibit LPL is lower compared to the activity of ANGPTL4 alone. This seems to indicate that ANGPTL8 is a natural inhibitor of ANGPTL4. When the stoichiometric ratio is about 1:1, ANGPTL8 has the most obvious inhibitory effects on ANGPTL4, and this ratio is close to the physiological ratio of these two proteins in the human body [29]. 


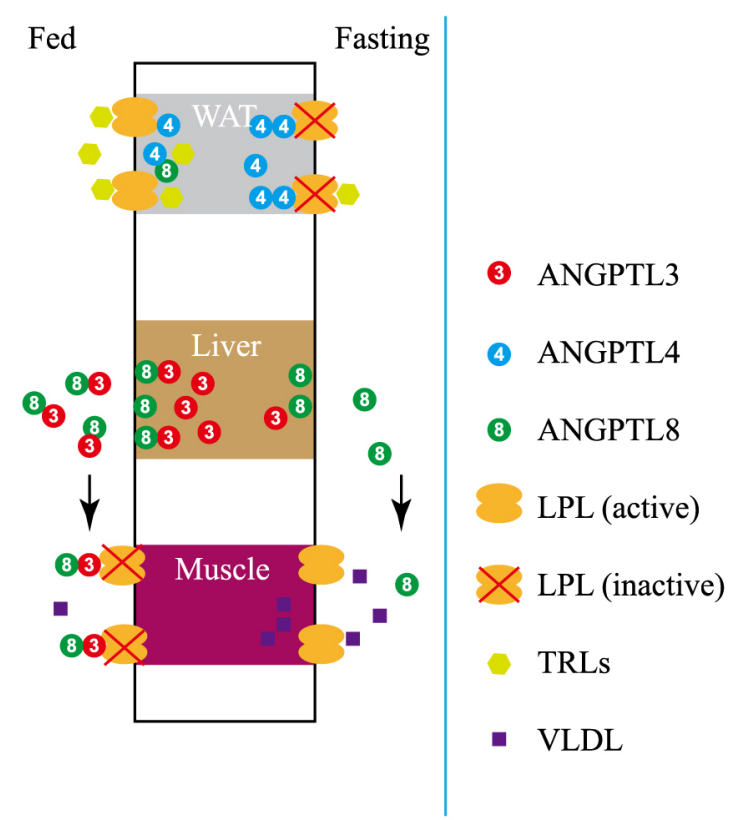

Fig. 3 Expression of ANGPTL3, 4, and 8 in white adipose tissue (WAT), liver, and muscle tissues under fasting/starvation and feeding status.

\section{The Differential Regulation of ANGPTL Proteins under Different Nutritional and Metabolic Status}

The expression levels of ANGPTLs are mainly regulated by the nutritional status of the body [21] (Fig. 3). In the case of starvation, the expression of ANGPTL3 and 8 decreases, and re-feeding leads to an increase in expressions of ANGPTL3 and 8 [23]. Conversely, the expression of ANGPTL4 in adipose tissue increases when fast, and the expression decreases after feeding [38].

\section{Starvation status}

Under starvation, the expression of ANGPTL8 decreased by nearly $70 \%$ in WAT [21], and the expression of ANGPTL8 in BAT and liver was also significantly reduced [39]. Under starvation, circulating ANGPTL8/ANGPTL3 complex reduces [10], which increases LPL activity in peripheral tissues and in turns increases the uptake of VLDL by skeletal muscles and the heart. These processes ensure the energy supply to maintain the physiological functions of the body. On the contrary, fasting caused up-regulation of ANGPTL4 in WAT [21, 39]. The up-regulated ANGPTL4 reduces the uptake of circulating triglycerides by WAT through reducing LPL activity. In addition, up-regulated ANGPTL4 also increases the breakdown of white fat and secretion of fatty acids for energy supply [40].

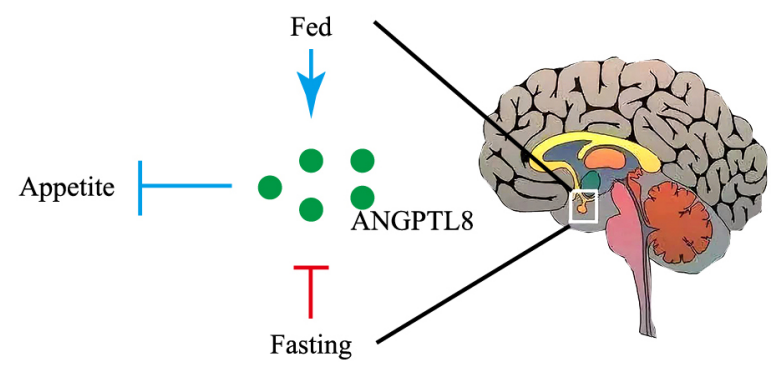

Fig. 4 Effects of nutritional signals on the expression of ANGPTL8 which regulates the feeding behavior in Hypothalamus.

\section{Feeding status}

Diet can increase the expression of ANGPTL8 in liver, and also increase the amounts of ANGPTL3, ANGPTL8, and ANGPTL3/8 complex in circulation. Increased ANGPTL3/8 complex in circulation inhibits the activity of LPL, and therefore the amounts of skeletal muscles and heart to absorb VLDL are reduced [10]. Therefore, energy is stored after eating. Studies have shown that X receptor alpha $(\mathrm{LXR} \alpha)$ in liver plays an important role in the up-regulation of ANGPTL8 induced by feeding [23]. The expression of ANGPTL4 in white adipose tissue was down-regulated in abdominally obese subjects [38]. The increased expression of ANGPTL8 may form more ANGPTL4/ANGPTL8 complexes with ANGPTL4, thus reducing the inhibitory activity of the complexes [29]. Therefore, LPL activity increased in white adipose tissue, and triglyceride uptake increased in white adipose tissue. All these processes increased the storage and accumulation of energy.

\section{ANGPTL8 participates in appetite regulation}

The study showed that the ANGPTL8 levels in plasma in anorexia patients were higher than those in the normal control group [41]. Central or peripheral ANGPTL8 intervention significantly reduced food intake in mice. The hypothalamus has long been considered as a control center for regulating energy balance. ANGPTL8 is widely expressed in appetite-related nuclei, including paraventricular nucleus (PVN), dorsomedial hypothalamus (DMH), ventromedial hypothalamus, and hypothalamic arcuate nucleus (ARC) [42]. It has been indicated that hunger and satiety signals regulate the levels of ANGPTL8 and LPL is highly expressed in the brain. The information suggests that ANGPTL8 regulates the activity of LPL in brain, and ultimately regulates feeding behavior through hypothalamus (Fig. 4).

\section{Cold stimulation}

In response to cold stimulation, brown adipose tissue 
produces heat. The expression of ANGPTL8 in BAT was up-regulated and ANGPTL4 was down-regulated at cold stimulation $\left(4^{\circ} \mathrm{C}, 4\right.$ hours) [43]. In wild-type mice, cold stimulation can increase LPL-mediated degradation of triglyceride-rich lipoproteins (TRLs) in BAT to produce fatty acids for tremor heat production. The expression of ANGPTL8 increased when WAT is turned into BAT [44]. In addition, long-term ANGPTL8 exposure can increase the expression of BAT proteins such as UCP1, TMEM26, TBX1, and b3-AR during differentiation of hASC in vitro. These results suggest that ANGPTL8 may be involved in the regulation of lipid browning.

\section{ANGPTL8 and Metabolic Diseases}

Studies have shown that ANGPTL8 participates in lipid metabolism. It was reported that in diabetic patients, ANGPTL8 levels were significantly positively correlated with triglyceride (TG) and LDL-C levels, and negatively correlated with HDL-C levels [45, 46]. Javier et al. found that the circulating ANGPTL8 levels were significantly lower in patients with dyslipidemia characterized by low HDL-C or high TG levels [47]. A crosssectional study of Chinese population showed that circulating levels of ANGPTL8 in full-length were significantly increased in patients with dyslipidemia compared with those without dyslipidemia. The circulating levels of ANGPTL8 in full-length were positively correlated with serum non-HDL-C, TG and TC levels, but negatively correlated with HDL-C levels [48]. The discrepancy among these results may be due to different blood glucose levels and diverse types of kits used. In addition, non-HDL-C is considered to be a strong predictor of the total number of atherogenic lipoprotein particles [49], indicating the total cholesterol of apolipoprotein B containing lipoproteins, including TG rich lipoproteins (very low density lipoproteins, medium density lipoproteins, and residual lipoproteins) [50].

In addition to lipid metabolism, ANGPTL8 may play a role in glucose metabolism by improving insulin resistance [19]. Under physiological status, the knockout of ANGPTL8 had no significant effects on glucose metabolism in mice [12]. In HEPG2 cells with insulin resistance, ANGPTL8 can increase glycogen synthesis and inhibit hepatic gluconeogenesis. Recently, ANGPTL8 has been widely investigated for its ability to promote the proliferation of $\beta$ cells, but the results were eventually disagreed by several other paper [12, 51-54]. Although ANGPTL8 is not involved in the regulation of $\beta$ cell proliferation, its correlation with endocrine and metabolic diseases, such as diabetes mellitus, has attracted much attention. Espes et al. observed an increase of circulating ANGPTL8 in patients with long-term type 1 diabetes mellitus [55]. Fu et al. also observed an increase in ANGPTL8 level in type 2 diabetic patients [56]. Our previous studies also showed that circulating ANGPTL8 levels in newly diagnosed type 2 diabetic patients increased by about two times [57]. Generally speaking, many studies have shown that levels of ANGPTL8 change in polycystic ovary syndrome (PCOS) [58, 59], metabolic syndrome $[60,61]$, non-alcoholic fatty liver [62, 63], hypothyroidism [64], Graves' disease [65], and coronary heart disease [66].

\section{Conclusion}

ANGPTL3, ANGPTL4, and ANGPTL8 can all inhibit the activity of LPL by affecting the stability of LPL dimers. But their inhibition capability and tissue specificity are different and ANGPTL4 responds to nutrients opposingly compared to the other two. Generally speaking, ANGPTL8 is mainly expressed in liver and adipose tissues, ANGPTL3 is mainly expressed in liver, and ANGPTL4 is mainly expressed in adipose tissues. Feeding inhibits LPL activity in heart and skeletal muscles and causes WAT storage of circulating triglycerides through activating ANGPTL8/ANGPTL3 pathway. On the contrary, fasting inhibits LPL activity in WAT and induces oxidation of triglyceride in heart and skeletal muscles through activating ANGPTL4. In sum, this review discusses the interaction among ANGPTL3, 4, 8 proteins, and summarizes the relationship between the expression of these proteins and lipid metabolism under different nutritional and metabolic status.

\section{Acknowledgements}

This study was supported by the National Natural Science Foundation of China (81870548, 81570721, 31471294 and 31771521), the Social Development Project of Jiangsu Province (BE2018692, BE2016718), the Natural Science Foundation of Jiangsu Province, China (BK20151331, BK20191222), the High Caliber Medical Personnel Foundation of Jiangsu Province (LGY2016053), the Six Talent Peaks Project in Jiangsu Province (2015-WSN-006, 2016-SWYY-011), the Scientific Research Projects of Jiangsu Health and Family Planning Commission (Y2018109), Start-up Scientific Research Fund for the Returned Oversea Scholars from Chinese Ministry of Education, and Top Talent of Innovative Research Team of Jiangsu Province.

\section{Disclosure}

The authors have no conflicts of interest to declare. 


\section{References}

1. Armand M (2007) Lipases and lipolysis in the human digestive tract: where do we stand? Curr Opin Clin Nutr Metab Care 10: 156-164.

2. Wilde PJ, Chu BS (2011) Interfacial \& colloidal aspects of lipid digestion. Adv Colloid Interface Sci 165: 14-22.

3. Li C (2006) Genetics and regulation of angiopoietin-like proteins 3 and 4. Curr Opin Lipidol 17: 152-156.

4. Mead JR, Irvine SA, Ramji DP (2002) Lipoprotein lipase: structure, function, regulation, and role in disease. $J \mathrm{Mol}$ Med (Berl) 80: 753-769.

5. Zhang R, Abou-Samra AB (2014) A dual role of lipasin (betatrophin) in lipid metabolism and glucose homeostasis: consensus and controversy. Cardiovasc Diabetol 13: 133.

6. Wang H, Eckel RH (2009) Lipoprotein lipase: from gene to obesity. Am J Physiol Endocrinol Metab 297: E271E288.

7. Mysling S, Kristensen KK, Larsson M, Beigneux AP, Gardsvoll H, et al. (2016) The acidic domain of the endothelial membrane protein GPIHBP1 stabilizes lipoprotein lipase activity by preventing unfolding of its catalytic domain. Elife 5: e12095.

8. Dijk W, Kersten S (2016) Regulation of lipid metabolism by angiopoietin-like proteins. Curr Opin Lipidol 27: 249 256.

9. Peloso GM, Auer PL, Bis JC, Voorman A, Morrison AC, et al. (2014) Association of low-frequency and rare coding-sequence variants with blood lipids and coronary heart disease in 56,000 whites and blacks. Am J Hum Genet 94: 223-232.

10. Quagliarini F, Wang Y, Kozlitina J, Grishin NV, Hyde R, et al. (2012) Atypical angiopoietin-like protein that regulates ANGPTL3. Proc Natl Acad Sci U S A 109: 1975119756.

11. Yoshida K, Shimizugawa T, Ono M, Furukawa H (2002) Angiopoietin-like protein 4 is a potent hyperlipidemiainducing factor in mice and inhibitor of lipoprotein lipase. J Lipid Res 43: 1770-1772.

12. Wang Y, Quagliarini F, Gusarova V, Gromada J, Valenzuela DM, et al. (2013) Mice lacking ANGPTL8 (Betatrophin) manifest disrupted triglyceride metabolism without impaired glucose homeostasis. Proc Natl Acad Sci US A 110: 16109-16114.

13. Ren G, Kim JY, Smas CM (2012) Identification of RIFL, a novel adipocyte-enriched insulin target gene with a role in lipid metabolism. Am J Physiol Endocrinol Metab 303: E334-E351.

14. Fu Z, Yao F, Abou-Samra AB, Zhang R (2013) Lipasin, thermoregulated in brown fat, is a novel but atypical member of the angiopoietin-like protein family. Biochem Biophys Res Commun 430: 1126-1131.

15. Santulli G (2014) Angiopoietin-like proteins: a comprehensive look. Front Endocrinol (Lausanne) 5: 4.

16. Hato T, Tabata M, Oike Y (2008) The role of angiopoietin-like proteins in angiogenesis and metabo- lism. Trends Cardiovasc Med 18: 6-14.

17. Siddiqa A, Ahmad J, Ali A, Paracha RZ, Bibi Z, et al. (2016) Structural characterization of ANGPTL8 (betatrophin) with its interacting partner lipoprotein lipase. Comput Biol Chem 61: 210-220.

18. DiStefano JK (2019) Angiopoietin-like 8 (ANGPTL8) expression is regulated by miR-143-3p in human hepatocytes. Gene 681: 1-6.

19. Rong Guo X, Li Wang X, Chen Y, Hong Yuan Y, Mei Chen Y, et al. (2016) ANGPTL8/betatrophin alleviates insulin resistance via the Akt-GSK3 $\beta$ or Akt-FoxO1 pathway in HepG2 cells. Exp Cell Res 345: 158-167.

20. Liu J, Yang K, Xiao W, Le Y, Lang S, et al. (2018) GLP-1 receptor agonists stimulate ANGPTL8 production through the PI3K/Akt pathway in a GLP-1 receptor-dependent manner. Peptides 106: 83-90.

21. Zhang R (2012) Lipasin, a novel nutritionally-regulated liver-enriched factor that regulates serum triglyceride levels. Biochem Biophys Res Commun 424: 786-792.

22. Stephens JM (2012) RIFL aims to be a new player in lipid metabolism. Am J Physiol Endocrinol Metab 303: E332E333.

23. Dang F, Wu R, Wang P, Wu Y, Azam MS, et al. (2016) Fasting and feeding signals control the oscillatory expression of Angptl8 to modulate lipid metabolism. Sci Rep 6: 36926.

24. Luo D, Chen X, Yang W, Ran W, Wen Z (2018) Angiopoietin-like 8 improves insulin resistance and attenuates adipose tissue inflammation in diet-induced obese mice. Exp Clin Endocrinol Diabetes.

25. Zhang Y, Guo X, Yan W, Chen Y, Ke M, et al. (2017) ANGPTL8 negatively regulates NF-kappaB activation by facilitating selective autophagic degradation of IKKgamma. Nat Commun 8: 2164.

26. Mysore R, Liebisch G, Zhou Y, Olkkonen VM, Nidhina Haridas PA (2017) Angiopoietin-like 8 (Angpt18) controls adipocyte lipolysis and phospholipid composition. Chem Phys Lipids 207: 246-252.

27. Shimamura M, Matsuda M, Kobayashi S, Ando Y, Ono M, et al. (2003) Angiopoietin-like protein 3, a hepatic secretory factor, activates lipolysis in adipocytes. Biochem Biophys Res Commun 301: 604-609.

28. Chi X, Britt EC, Shows HW, Hjelmaas AJ, Shetty SK, et al. (2017) ANGPTL8 promotes the ability of ANGPTL3 to bind and inhibit lipoprotein lipase. Mol Metab 6: 11371149 .

29. Kovrov O, Kristensen KK, Larsson E, Ploug M, Olivecrona G (2019) On the mechanism of angiopoietinlike protein 8 for control of lipoprotein lipase activity. $J$ Lipid Res 60: 783-793.

30. Haller JF, Mintah IJ, Shihanian LM, Stevis P, Buckler D, et al. (2017) ANGPTL8 requires ANGPTL3 to inhibit lipoprotein lipase and plasma triglyceride clearance. J Lipid Res 58: 1166-1173.

31. Kersten S, Mandard S, Tan NS, Escher P, Metzger D, et 
al. (2000) Characterization of the fasting-induced adipose factor FIAF, a novel peroxisome proliferator-activated receptor target gene. J Biol Chem 275: 28488-28493.

32. Yoon JC, Chickering TW, Rosen ED, Dussault B, Qin Y, et al. (2000) Peroxisome proliferator-activated receptor gamma target gene encoding a novel angiopoietin-related protein associated with adipose differentiation. Mol Cell Biol 20: 5343-5349.

33. Chi X, Shetty SK, Shows HW, Hjelmaas AJ, Malcolm EK, et al. (2015) Angiopoietin-like 4 modifies the interactions between lipoprotein lipase and its endothelial cell transporter GPIHBP1. J Biol Chem 290: 11865-11877.

34. Dijk W, Beigneux AP, Larsson M, Bensadoun A, Young $\mathrm{SG}$, et al. (2016) Angiopoietin-like 4 promotes intracellular degradation of lipoprotein lipase in adipocytes. $J$ Lipid Res 57: 1670-1683.

35. Dijk W, Ruppert PMM, Oost LJ, Kersten S (2018) Angiopoietin-like 4 promotes the intracellular cleavage of lipoprotein lipase by PCSK3/furin in adipocytes. $J$ Biol Chem 293: 14134-14145.

36. Dijk W, Heine M, Vergnes L, Boon MR, Schaart G, et al. (2015) ANGPTL4 mediates shuttling of lipid fuel to brown adipose tissue during sustained cold exposure. Elife 4: $\mathrm{e} 08428$.

37. Ge H, Yang G, Yu X, Pourbahrami T, Li C (2004) Oligomerization state-dependent hyperlipidemic effect of angiopoietin-like protein 4. J Lipid Res 45: 2071-2079.

38. Dijk W, Schutte S, Aarts EO, Janssen IMC, Afman L, et al. (2018) Regulation of angiopoietin-like 4 and lipoprotein lipase in human adipose tissue. J Clin Lipidol 12: 773-783.

39. Larsson M, Allan CM, Heizer PJ, Tu Y, Sandoval NP, et al. (2018) Impaired thermogenesis and sharp increases in plasma triglyceride levels in GPIHBP1-deficient mice during cold exposure. J Lipid Res 59: 706-713.

40. Cushing EM, Chi X, Sylvers KL, Shetty SK, Potthoff MJ, et al. (2017) Angiopoietin-like 4 directs uptake of dietary fat away from adipose during fasting. Mol Metab 6: 809818.

41. Barja-Fernandez S, Folgueira C, Seoane LM, Casanueva FF, Dieguez C, et al. (2015) Circulating betatrophin levels are increased in anorexia and decreased in morbidly obese women. J Clin Endocrinol Metab 100: E1188-E1196.

42. Wang R, Yuan J, Zhang C, Wang L, Liu Y, et al. (2018) Neuropeptide Y-positive neurons in the dorsomedial hypothalamus are involved in the anorexic effect of Angpt18. Front Mol Neurosci 11: 451.

43. Fu Z, Yao F, Abou-Samra AB, Zhang R (2013) Lipasin, thermoregulated in brown fat, is a novel but atypical member of the angiopoietin-like protein family. Biochem Biophys Res Commun 430: 1126-1131.

44. Martinez-Perez B, Ejarque M, Gutierrez C, Nunez-Roa C, Roche K, et al. (2016) Angiopoietin-like protein 8 (ANGPTL8) in pregnancy: a brown adipose tissue-derived endocrine factor with a potential role in fetal growth. Transl Res 178: 1-12.

45. Chen CC, Susanto H, Chuang WH, Liu TY, Wang CH
(2016) Higher serum betatrophin level in type 2 diabetes subjects is associated with urinary albumin excretion and renal function. Cardiovasc Diabetol 15: 3.

46. Ghasemi H, Tavilani H, Khodadadi I, Saidijam M, Karimi J (2015) Circulating betatrophin levels are associated with the lipid profile in type 2 diabetes. Chonnam Med $J$ 51: 115-119.

47. Gómez-Ambrosi J, Pascual-Corrales E, Catalán V, Rodríguez A, Ramírez B, et al. (2016) Altered concentrations in dyslipidemia evidence a role for ANGPTL8/betatrophin in lipid metabolism in humans. $J$ Clin Endocrinol Metab 101: 3803-3811.

48. Yang S, Jiao X, Huo X, Zhu M, Wang Y, et al. (2018) Association between circulating full-length angiopoietinlike protein 8 and non-high-density lipoprotein cholesterol levels in Chinese non-diabetic individuals: a crosssectional study. Lipids Health Dis 17: 161.

49. Liao P, Zeng R, Zhao X, Guo L, Zhang M (2017) Prognostic value of non-high-density lipoprotein cholesterol for mortality in patients with coronary heart disease: a systematic review and meta-analysis. Int J Cardiol 227: 950955.

50. Nordestgaard BG, Langsted A, Mora S, Kolovou G, Baum $\mathrm{H}$, et al. (2016) Fasting is not routinely required for determination of a lipid profile: clinical and laboratory implications including flagging at desirable concentration cut-points-a joint consensus statement from the European Atherosclerosis Society and European Federation of Clinical Chemistry and Laboratory Medicine. Eur Heart $J$ 37: 1944-1958.

51. Abu-Farha M, Al Madhoun A, Abubaker J (2016) The rise and the fall of betatrophin/ANGPTL8 as an inducer of $\beta$ Cell proliferation. J Diabetes Res 2016: 4860595.

52. Jiao Y, Le Lay J, Yu M, Naji A, Kaestner KH (2014) Elevated mouse hepatic betatrophin expression does not increase human beta-cell replication in the transplant setting. Diabetes 63: 1283-1288.

53. Gusarova V, Alexa CA, Na E, Stevis PE, Xin Y, et al. (2014) ANGPTL8/betatrophin does not control pancreatic beta cell expansion. Cell 159: 691-696.

54. Yi P, Park JS, Melton DA (2017) Retraction notice to: betatrophin: a hormone that controls pancreatic beta cell proliferation. Cell 168: 326.

55. Espes D, Lau J, Carlsson PO (2014) Increased circulating levels of betatrophin in individuals with long-standing type 1 diabetes. Diabetologia 57: 50-53.

56. Fu Z, Berhane F, Fite A, Seyoum B, Abou-Samra AB, et al. (2014) Elevated circulating lipasin/betatrophin in human type 2 diabetes and obesity. Sci Rep 4: 5013.

57. Hu H, Sun W, Yu S, Hong X, Qian W, et al. (2014) Increased circulating levels of betatrophin in newly diagnosed type 2 diabetic patients. Diabetes Care 37: 27182722.

58. Li L, Zhang F, Cui J, Shi Y, Xiang J, et al. (2017) Association of betatrophin with metabolic characteristics in overweight/obese and lean women with PCOS. Gynecol Endocrinol 33: 238-243. 
59. Calan M, Yilmaz O, Kume T, Unal Kocabas G, Yesil Senses P, et al. (2016) Elevated circulating levels of betatrophin are associated with polycystic ovary syndrome. Endocrine 53: 271-279.

60. Abu-Farha M, Abubaker J, Al-Khairi I, Cherian P, Noronha F, et al. (2016) Circulating angiopoietin-like protein 8 (betatrophin) association with HsCRP and metabolic syndrome. Cardiovasc Diabetol 15: 25.

61. Wang H, Lai Y, Han C, Liu A, Fan C, et al. (2016) The effects of serum ANGPTL8/betatrophin on the risk of developing the metabolic syndrome - a prospective study. Sci Rep 6: 28431.

62. Lee YH, Lee SG, Lee CJ, Kim SH, Song YM, et al. (2016) Association between betatrophin/ANGPTL8 and non-alcoholic fatty liver disease: animal and human studies. Sci Rep 6: 24013.
63. Cengiz M, Ozenirler S, Kocabiyik M (2016) Serum betatrophin level as a new marker for noninvasive assessment of nonalcoholic fatty liver disease and liver fibrosis. Eur $J$ Gastroenterol Hepatol 28: 57-63.

64. Han C, Xia X, Liu A, Zhang X, Zhou M, et al. (2016) Circulating betatrophin is increased in patients with overt and subclinical hypothyroidism. Biomed Res Int 2016: 5090852.

65. Li H, Xu M, Zhao L, Xia H, Li Y, et al. (2019) Decreased circulating levels of ANGPTL8 in Graves' disease patients. Hormones (Athens) 18: 189-195.

66. Jiao X, He J, Yang Y, Yang S, Li J, et al. (2018) Associations between circulating full-length angiopoietin-like protein 8 levels and severity of coronary artery disease in Chinese non-diabetic patients: a case-control study. Cardiovasc Diabetol 17: 92. 